\title{
Functional monocytic myeloid-derived suppressor cells increase in blood but not airways and predict COVID-19 severity
}

\author{
Sara Falck-Jones, ${ }^{1}$ Sindhu Vangeti, ${ }^{1}$ Meng Yu, ${ }^{1}$ Ryan Falck-Jones, ${ }^{2,3}$ Alberto Cagigi, ${ }^{1}$ Isabella Badolati, ${ }^{1}$ Björn Österberg, ${ }^{1}$ \\ Maximilian Julius Lautenbach, ${ }^{4}$ Eric Åhlberg, ${ }^{1}$ Ang Lin, ${ }^{1,5}$ Rico Lepzien, ${ }^{1}$ Inga Szurgot, ${ }^{1}$ Klara Lenart, ${ }^{1}$ Fredrika Hellgren, ${ }^{1}$ \\ Holden Maecker, ${ }^{6}$ Jörgen Sälde, ${ }^{7}$ Jan Albert, ${ }^{8,9}$ Niclas Johansson, ${ }^{4,10}$ Max Bell, ${ }^{2,3}$ Karin Loré, ${ }^{1}$ Anna Färnert, ${ }^{4,10}$ \\ and Anna Smed-Sörensen ${ }^{1}$
}

'Division of Immunology and Allergy, Department of Medicine Solna, Karolinska Institutet, Karolinska University Hospital, Stockholm, Sweden. ${ }^{2}$ Department of Physiology and Pharmacology, Karolinska Institutet, Stockholm, Sweden. ${ }^{3}$ Department of Perioperative Medicine and Intensive Care, Karolinska University Hospital, Stockholm, Sweden. ${ }^{4}$ Division of Infectious Diseases, Department of Medicine Solna, Center for Molecular Medicine, Karolinska Institutet, Stockholm, Sweden. ${ }^{5}$ Stemirna Therapeutics Inc., Shanghai, China. ${ }^{6}$ Stanford University Medical Center, Stanford, California, USA. ${ }^{7}$ Health Care Services Stockholm County (SLSO), Stockholm, Sweden. ${ }^{8}$ Department of Microbiology, Tumor and Cell Biology, Karolinska Institutet, Stockholm, Sweden. ${ }^{9}$ Department of Clinical Microbiology and ${ }^{10}$ Department of Infectious Diseases, Karolinska University Hospital Solna, Stockholm, Sweden.

\begin{abstract}
The immunopathology of coronavirus disease 2019 (COVID-19) remains enigmatic, causing immunodysregulation and T cell lymphopenia. Monocytic myeloid-derived suppressor cells (M-MDSCs) are T cell suppressors that expand in inflammatory conditions, but their role in acute respiratory infections remains unclear. We studied the blood and airways of patients with COVID-19 across disease severities at multiple time points. M-MDSC frequencies were elevated in blood but not in nasopharyngeal or endotracheal aspirates of patients with COVID-19 compared with healthy controls. M-MDSCs isolated from patients with COVID-19 suppressed T cell proliferation and IFN- $\gamma$ production partly via an arginase 1-dependent (Arg-1-dependent) mechanism. Furthermore, patients showed increased Arg-1 and IL-6 plasma levels. Patients with COVID-19 had fewer T cells and downregulated expression of the $\operatorname{CD} 3 \zeta$ chain. Ordinal regression showed that early M-MDSC frequency predicted subsequent disease severity. In conclusion, M-MDSCs expanded in the blood of patients with COVID-19, suppressed T cells, and were strongly associated with disease severity, indicating a role for M-MDSCs in the dysregulated COVID-19 immune response.
\end{abstract}

\section{Introduction}

The pathogenesis of coronavirus disease 2019 (COVID-19) caused by severe acute respiratory syndrome coronavirus 2 (SARS-CoV-2) remains elusive. SARS-CoV-2 infection ranges from asymptomatic disease to multiorgan failure and death (1). COVID-19 is characterized by influenza-like symptoms (including fever, cough, and myalgia) and, in severe cases, respiratory failure and acute respiratory distress syndrome, occurring in approximately $40 \%$ of hospitalized patients (1-3). Fatal COVID-19 is caused by tissue-directed immunopathology, especially in the lungs, rather than by the virus itself $(4,5)$. Furthermore, it is known that immune cells differ depending on their anatomical location (6-10). Therefore, studying both systemic and respiratory immune responses in COVID-19 is important to fully understand its pathogenesis and to identify the factors that dictate disease severity.

COVID-19 is associated with substantial immune activation including elevated levels of proinflammatory cytokines such as

Conflict of interest: The authors have declared that no conflict of interest exists. Copyright: @ 2021, American Society for Clinical Investigation.

Submitted: September 29, 2020; Accepted: January 13, 2021; Published: March 15, 2021 Reference information: J Clin Invest. 2021;131(6):e144734.

https://doi.org/10.1172/JCl144734.
IL-6 (11). Furthermore, T cell lymphopenia occurs, especially in critical cases (12), but the underlying mechanisms remain unclear. SARS-CoV-2-specific T cells are important in combating the virus (13), and a functional T cell response is critical for clearing infections in general. Myeloid-derived suppressor cells (MDSCs) are myeloid immune cells with an immature phenotype and a potent $\mathrm{T}$ cell-suppressive capacity (14-16). MDSCs expand in inflammatory conditions including cancer, autoimmune diseases, and chronic viral infections like HIV and hepatitis C (17). Two subpopulations of MDSCs have been identified on the basis of their phenotypic and morphological features: monocytic MDSCs (M-MDSCs) and polymorphonuclear MDSCs (PMN-MDSCs), with partly overlapping functions $(18,19)$. MDSC-driven mechanisms of T cell suppression include the secretion of arginase 1 (Arg-1), thereby catabolizing L-arginine, the generation of ROS and NO, the direct engagement of $\mathrm{T}$ cell inhibitory and apoptotic receptors, and the production of inhibitory cytokines such as IL-10 and TGF- $\beta$ (20).

Single-cell RNA-Seq, mass cytometry, and flow cytometry on blood have suggested that expansion of suppressive myeloid cells is a hallmark of severe COVID-19 $(21,22)$. Furthermore, a high frequency of PMN-MDSCs was recently reported to correlate with disease severity in COVID-19 (23). However, functional and mechanistic data on the role of M-MDSCs during COVID-19 are 
lacking, and this is further confounded by the lack of knowledge of the role of M-MDSCs in respiratory infections in general.

In this study, we investigated M-MDSCs in patients with COVID-19 across disease severities and compared these cells with those in patients with influenza and in healthy controls (HCs). Influenza A virus infection is often compared with COVID-19 because of similarities including the diverse clinical presentation and the route of transmission $(24,25)$. We found a striking association between the frequency of blood M-MDSCs and COVID-19 disease severity. However, the frequency of M-MDSCs from the nasopharynx and lower airways did not correlate with disease severity in COVID-19. Importantly, purified M-MDSCs were functional and suppressed $\mathrm{T}$ cell proliferation, partly via an Arg-1-dependent mechanism. In line with this, plasma Arg-1 levels were elevated in patients with COVID-19 in a disease severity-dependent manner. Finally, we found that early blood M-MDSC frequency predicted subsequent disease severity, suggesting both that M-MDSCs are involved in the dysregulation of the immune response in COVID-19 and that they may be used as a potential prognostic marker in patients with COVID-19.

\section{Results}

Study participant characteristics. In total, 147 adults with PCR-confirmed SARS-CoV-2 infection, ranging from mild to fatal disease, were enrolled in the study: 91 patients from hospital wards, 43 patients from the intensive care unit (ICU), 3 patients from an outpatient clinic, and 10 household contacts, and blood as well as respiratory samples were collected longitudinally (Figure 1A and Supplemental Figure 1; supplemental material available online with this article; https://doi.org/10.1172/JCI144734DS1). Identical samples from 44 patients with PCR-confirmed influenza A virus infection with mild-to-moderate disease, as well as from 33 agematched HCs, were included for comparison (Figure 1B and Table 1). As expected, disease severity in the COVID-19 patient cohort varied over time (Figure 1C, Table 2, and Supplemental Figure 1), and some patients deteriorated during their hospital stay. At peak disease severity, $13 \%$ of patients were classified as having mild disease, $39 \%$ as moderate, and $39 \%$ as severe (Figure 1C). Furthermore, there were 12 recorded fatalities (8.1\%) in the COVID-19 cohort during the observation period (Figure 1C). The peak disease severity score prior to death was 6 in all but 2 patients, who had scores of 4 and 5 , respectively. At the end of the study period, 110 of the nonfatal, hospitalized patients had been discharged, while 12 patients remained hospitalized, all of whom were already classified as having severe disease. The distribution of age varied significantly across peak disease severity groups in patients with COVID-19 $(P<0.001)$, as did BMI $(P<0.001)$, male sex $(P=0.004)$, and the Charlson comorbidity index (CCI) $(P=0.042$ and Table 2$)$.

$M-M D S C$ frequencies are elevated in blood from COVID-19 and influenza patients, proportional to disease severity. To investigate the dynamics of M-MDSCs during COVID-19 disease, we performed an extensive analysis of samples from patients with COVID-19 across disease severity and compared them with samples from patients with influenza and HCs. PBMCs and cells from nasopharyngeal aspirates (NPAs) and endotracheal aspirates (ETAs) were stained and analyzed by flow cytometry. We identified M-MDSCs as CD14 ${ }^{+}$ cells within the lineage-negative (CD3- ${ }^{-}$D56 $\left.{ }^{-} \mathrm{CD} 19^{-} \mathrm{CD} 2 \mathrm{O}^{-} \mathrm{CD} 66^{-}\right)$, HLA-DR- population (Figure $2 \mathrm{~A}$ ). In blood, we found that the peak frequency of M-MDSCs was significantly increased in both patients with COVID-19 and those with influenza compared with HCs (Figure 2B). The frequency of M-MDSCs in NPAs had a higher spread among both HCs and patients compared with the M-MDSC frequencies in blood (Figure 2B). Albeit in a small number of patients with mild-to-moderate disease, patients with influenza displayed a clear pattern of elevated frequencies of M-MDSCs in NPAs as compared with patients with COVID-19 and HCs. The elevated frequency of M-MDSCs in NPAs in influenza patients compared with COVID-19 patients was also evident when comparing only mild and moderate influenza and COVID-19 cases $(P=0.0016$; data not shown). In contrast to NPAs, patients with COVID-19 with more severe disease had significantly higher peak M-MDSC frequencies in the blood, whereas COVID-19 patients with mild disease had blood M-MDSC frequencies comparable to those seen in HCs (Figure 2C). The frequency of M-MDSC in blood from COVID-19 patients seemed to decrease over time (Figure 2D) and returned to frequencies similar to those seen in HCs in follow-up samples taken during convalescence (33-65 days after study inclusion, Figure 2E).

Somewhat surprisingly, patients with COVID-19, in contrast to those with influenza, had low M-MDSC frequencies in NPAs, even when comparing patients with similar disease severities (Figure 2B). Since COVID-19 patients on average were included in the study and sampled significantly later after symptom onset compared with the influenza patients ( 18 vs. 5 days, respectively), we speculated that the infection-induced inflammation, including infiltration of M-MDSCs, might have transitioned into the lower airways in the patients with COVID-19 compared with those with influenza. To address this, we assessed whether M-MDSCs were present in ETAs from the lower airways in 20 patients with COVID-19 who were intubated. However, we did not find the frequency of M-MDSCs in ETAs to be elevated compared with NPAs from the same patients (Figure 2F). Although the levels of blood M-MDSCs in COVID-19 patients were significantly elevated compared with levels in HCs, the M-MDSC phenotype with respect to expression levels of CD62L, CCR2, and CD86 were similar (Figure 2G and data not shown). However, respiratory M-MDSCs expressed significantly lower levels of CD62L, CD86, and CCR2 compared with blood M-MDSCs, except for the upregulation of CCR2 on ETA M-MDSCs, (Figure 2, G-I).

Immunomodulatory treatment could be a possible confounder when studying immune cells including M-MDSCs. During the sampling period, cortisone was administered to 22 patients (Table 2), three of whom had ongoing treatment for other conditions before hospital admission. The M-MDSC frequencies among these patients were not significantly different compared with patients without cortisone treatment (Supplemental Figure 2). None of the patients received IL-6 inhibitors (tocilizumab) or IL-1 inhibitors during the sampling period. Ten patients received chloroquine phosphate.

The levels of PMN-MDSCs were analyzed in PBMCs from COVID-19 patients and HCs. We identified PMN-MDSCs as CD56CD14-CD3-CD19-HLA-DR ${ }^{-}$cells expressing CD66abce and lectin-like oxidized LDL receptor 1 (LOX-1) (26). We detected a dramatic increase in the frequency of PMN-MDSCs, with a clear association with disease severity (Figure 2J). As with M-MDSCs, the levels of PMN-MDSCs decreased over time in 6 patients from whom follow-up samples were collected (Figure $2 \mathrm{~K}$ ). Interestingly, the frequency of 
A
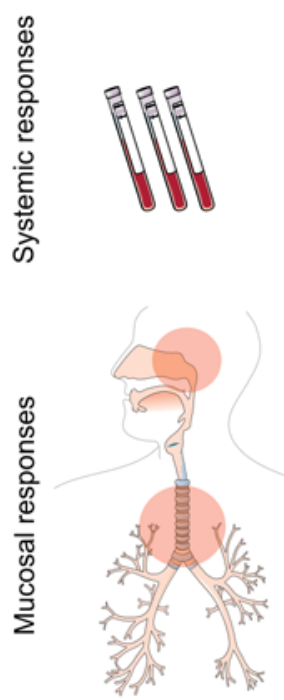

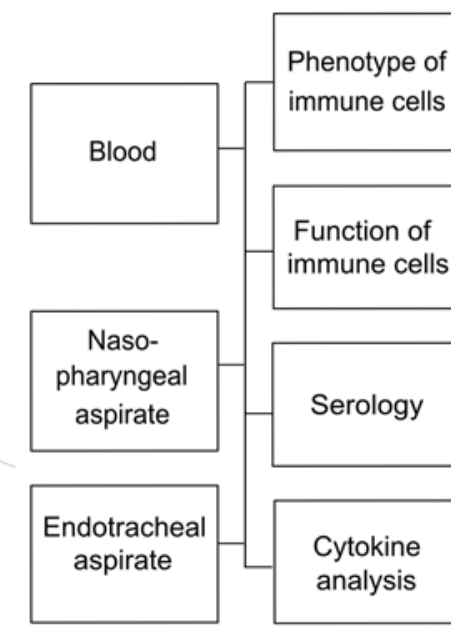

B

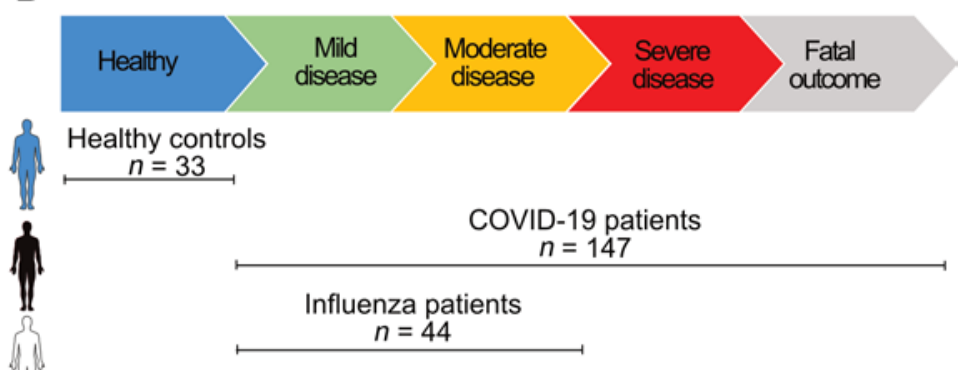

COVID-19 patients

Disease severity at inclusion

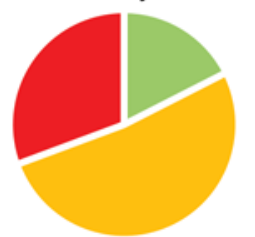

COVID-19 patients

Peak disease severity

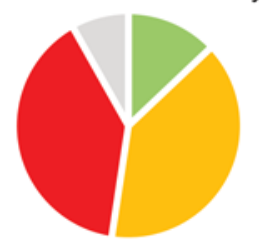

Figure 1. Study outline. (A) Blood and NPAs were collected from patients with COVID-19, patients with influenza, and HCs. ETAs were also collected from ICU patients. Cells were isolated from blood (PBMCs), NPAs, and ETAs and were analyzed fresh by flow cytometry and used for functional experiments. Aspirates and plasma were collected and used for serology and cytokine detection by ELISA. (B) Study participants were included and sampled across disease severities. Participants included HCs $(n=33)$, patients with mild or moderate influenza $(n=44)$, and patients with mild to fatal COVID-19 ( $n=147)$. (C) Pie charts show the distribution of disease severity at the time of study inclusion and the peak disease severity of patients with COVID-19. At the time of inclusion, $18 \%(n=24)$ had mild disease, mod52\% $(n=71)$ had moderate disease, and 31\% $(n=42)$ had severe disease. At peak disease severity, $13 \%(n=$ 19) had mild disease, $39 \%(n=58)$ had moderate disease, $39 \%(n=58)$ had severe disease, and $8 \%(n=12)$ had a fatal outcome.

CD16-expressing PMN-MDSCs was high, indicating a more mature PMN-MDSC phenotype (26-28), and this subset also increased in frequency with increasing disease severity (Supplemental Figure 3).

Altogether, these data indicate that severe COVID-19 disease is associated with elevated levels of M-MDSCs in the blood but not in the respiratory tract, at least at the time points studied.

M-MDSCs isolated from patients with COVID-19 suppress CD4 ${ }^{+}$ and $C D 8^{+} T$ cell proliferation. To functionally confirm the identity of M-MDSCs in patients with COVID-19, we evaluated their suppressive effect on T cells. Blood M-MDSCs from COVID-19 patients were purified and cocultured for 3 days with CFSE-labeled allogeneic PBMCs in the presence of staphylococcal enterotoxin B (SEB) (Supplemental Figure 4). As expected, SEB induced strong T cell proliferation (Figure 3A). However, the addition of M-MDSCs induced a significant suppression of both $\mathrm{CD}^{+}$and $\mathrm{CD} 8^{+} \mathrm{T}$ cell proliferation in a dose-dependent manner (Figure 3, A-C). In line with this, SEB induced high levels of IFN- $\gamma$ secretion that were significantly lower in cocultures with M-MDSCs present (Figure 3D). Arg-1 production is one effector mechanism by which M-MDSCs suppress T cell proliferation via the degradation of $\mathrm{L}$-arginine that is needed for proliferation. Indeed, the addition of L-arginine to the M-MDSC cocultures restored the concentration of IFN- $\gamma$ in the cell culture supernatants (Figure 3D). Furthermore, cocultures with M-MDSCs contained high levels of Arg-1, which was undetectable in cultures without M-MDSCs (Figure 3E). In cocultures supplemented with L-arginine, Arg-1 was no longer detectable, possibly due to complex formation of Arg- 1 and the substrate (ref. 29 and Figure 3E). Importantly, the addition of recombinant $\mathrm{L}$-arginine to the cocultures decreased the suppressive effect of M-MDSCs on T cells and partially restored $\mathrm{T}$ cell proliferation (Figure 3, F and G). This indicates that M-MDSCs from patients with COVID-19 use Arg-1 as one mechanism to suppress T cells. In conclusion, blood M-MDSCs isolated from patients with COVID-19 are functional and can suppress $\mathrm{T}$ cell proliferation and IFN- $\gamma$ secretion in a dose- and Arg-1-dependent manner.

Unfortunately, we were not able to assess the functional capacity of M-MDSCs isolated from patients with influenza A virus infection, as too few M-MDSCs could be isolated from the blood volumes obtained. This should be addressed in future studies.

M-MDSC-related cytokines are elevated in patients with COVID-19 and increase with disease severity. To further investigate the effect of elevated frequencies of blood M-MDSCs in patients with COVID-19, we measured cytokines that have been linked to M-MDSC function and activation in plasma and NPAs at the time of study inclusion. In plasma, patients with COVID-19 had significantly higher levels of Arg-1 than did HCs, but we observed no significant difference between COVID-19 patients and influenza patients (Figure 4A). Interestingly, Arg-1 levels in NPAs were higher than in plasma in all 3 groups, with no significant differences between the groups (Figure 4B). Among patients with COVID-19, plasma concentrations of Arg-1 were lower in those with mild disease compared with patients with moderate, severe, or fatal disease $(P=0.07, P=0.04$, and $P=0.01$, respectively; Figure $4 C)$.

Plasma concentrations of IL-6, a potent proinflammatory cytokine important for M-MDSC differentiation (14), were significantly increased in both COVID-19 patients and influenza patients compared with HCs (Figure 4D), whereas no statistically significant differences were observed in NPAs (Figure 4E). Furthermore, IL-6 levels were strikingly different across the COVID-19 disease severity groups (Figure 4F). Patients with mild COVID-19 had significantly lower IL-6 levels than did patients with moderate disease $(P=$ 
Table 1. Characteristics of the patients and HCs

\begin{tabular}{|c|c|c|c|c|}
\hline Cohort & COVID-19 & Influenza & HC & Significance $^{A}$ \\
\hline$n$ & 147 & 44 & 33 & \\
\hline Age in yr, mean (range) & $57(24-78)$ & $52(18-88)$ & $51(24-80)$ & $P \leq 0.05$ \\
\hline Males, $n(\%)$ & $109(74)$ & $19(43)$ & $23(70)$ & \\
\hline Females, $n(\%)$ & $38(26)$ & $25(57)$ & $10(30)$ & \\
\hline Onset to admission, days, mean (SD) & $10(5.8)$ & $5(3.2)$ & - & $P \leq 0.05$ \\
\hline Onset to inclusion, days, mean & $18(10)$ & $5(2)$ & - & $P<0.001$ \\
\hline \multicolumn{5}{|l|}{ Comorbidities } \\
\hline CCI, mean (SD) & $2(2)$ & $2(2)$ & - & NS \\
\hline BMI, mean (SD) & $28.5(4.6)$ & $26.7(4.9)$ & $25.0(3.3)$ & $P \leq 0.05$ \\
\hline Hypertension, $n(\%)$ & $55(38)$ & $14(33)$ & 0 & NS \\
\hline Diabetes, $n(\%)$ & $34(23)$ & $4(9.1)$ & 0 & NS \\
\hline Current smoker, $n$ (\%) & $10(7.0)$ & $7(16)$ & 0 & NS \\
\hline \multicolumn{5}{|l|}{ Laboratory analyses } \\
\hline $\operatorname{CRP}(\mathrm{mg} / \mathrm{L})$, mean $(\mathrm{SD})^{\mathrm{A}, \mathrm{B}}$ & 206 (134) & 119 (146) & $2(3)$ & $P<0.001$ \\
\hline WBC $\left(\times 10^{9} / L\right)$, mean $(S D)^{A, C}$ & $8.0(4.3)$ & $6.8(3.5)$ & $6.5(1.5)$ & NS \\
\hline Lymphocytes $\left(\times 10^{9} / L\right)$, mean $(S D)^{A, C}$ & $0.89(0.69)$ & $1.06(0.47)$ & $2.41(1.24)$ & $P<0.001$ \\
\hline Neutrophils $\left(\times 10^{9} / \mathrm{L}\right)$, mean $(\mathrm{SD})^{\mathrm{A}, \mathrm{C}}$ & $6.4(3.9)$ & $5.1(3.5)$ & $3.1(0.7)$ & $P<0.01$ \\
\hline Monocytes $\left(\times 10^{9} / \mathrm{L}\right)$, mean $(\mathrm{SD})^{\mathrm{A}, \mathrm{C}}$ & $0.44(0.27)$ & $0.64(0.35)$ & $0.54(0.24)$ & $P<0.01$ \\
\hline \multicolumn{5}{|l|}{ Outcome } \\
\hline Peak severity score ${ }^{0}$, mean (SD) & $4.49(1.65)$ & $1.80(0.82)$ & - & $P<0.001$ \\
\hline Fatal outcome, $n(\%)$ & $12(8.1)$ & 0 & 0 & \\
\hline \multicolumn{5}{|c|}{$\begin{array}{l}\text { AStatistical significance was determined by } 1 \text {-way ANOVA, Fisher's exact test, or Pearson's } \chi^{2} \\
\text { test. }{ }^{{ }^{2}} \text { Peak value. 'Value at maximum lymphopenia. DPeak severity score on a } 7 \text {-grade composite } \\
\text { scale (see Methods). CRP, C-reactive protein. Normal range: CRP }<3 \mathrm{mg} / \mathrm{L}, \mathrm{WBC} 3.5 \times 10^{9} / \mathrm{L} \text { to } 8.8 \\
\times 10^{9} / \mathrm{L} \text {, lymphocytes } 1.1 \times 10^{9} / \mathrm{L} \text { to } 3.5 \times 10^{9} / \mathrm{L} \text {, neutrophils } 1.6 \times 10^{9} / \mathrm{L} \text { to } 5.9 \times 10^{9} / \mathrm{L} \text {, monocytes } \\
0.2 \times 10^{9} / \mathrm{L} \text { to } 0.8 \times 10^{9} / \mathrm{L} \text {. }\end{array}$} \\
\hline
\end{tabular}

0.038), patients with severe disease $(P<0.001)$, and patients with a fatal outcome $(P \leq 0.01)$, respectively. COVID-19 patients with moderate disease also had significantly lower levels than did those with severe disease $(P=0.04)$. GM-CSF, which is important for M-MDSC development (14), was also measured in plasma but was only significantly elevated in patients with influenza (Figure 4G).

Several factors are involved in the generation of M-MDSCs, and it has been proposed that IFN- $\gamma$ is involved in the licensing process of monocyte differentiation into M-MDSCs (30). Therefore, we also measured IFN- $\gamma$ was in plasma from patients with COVID-19. However, in our cohort, we did not detect elevated IFN- $\gamma$ levels in patients compared with HCs (Supplemental Figure 5), and there was no association with disease severity.

Finally, we measured plasma concentrations of IL-10 and IL-1 $\beta$ in a subset of patients with COVID-19 who had moderate-to-fatal disease, but only IL-10 showed an association with disease severity (Figure 4, H and I). In summary, we found that cytokines involved in the activation and function of M-MDSCs were elevated in plasma from patients with COVID-19 and correlated with disease severity.

$T$ cells are reduced in the blood of patients with COVID-19 and have low CD3 $\zeta$ chain expression. Since M-MDSCs isolated from patients with COVID-19 efficiently suppressed T cells in vitro, we assessed the overall blood $\mathrm{T}$ cell frequency and function in the same patients using flow cytometry (Supplemental Figure 6). We found that the absolute numbers of peripheral blood CD $4^{+} \mathrm{T}$ cellswere decreased in the patients with COVID-19 with moderate, severe, or fatal disease compared with HCs $(P=0.02, P=0.001, P=$ 0.05 , respectively; Figure $5 \mathrm{~A})$. Similarly, the absolute numbers of $\mathrm{CD} 8^{+} \mathrm{T}$ cells were also significantly decreased in moderate, severe, and fatal COVID-19 disease compared with HCs $(P=0.004, P=0.005$, and $P=0.003$, respectively; Figure $5 \mathrm{~B})$. However, we found no correlation between blood M-MDSC frequencies and $\mathrm{T}$ cell counts in patients with COVID-19 (Figure 5C), either at peak or bottom frequencies or at any of the longitudinal time points studied in each patient (Figure 5C and data not shown).

We further investigated evidence of T cell suppression in patients with COVID-19 with varying disease severities, in patients with influenza, and in HCs by quantifying the expression of the CD3ל chain, a homodimer chain in the $\mathrm{T}$ cell receptor complex involved in $\mathrm{T}$ cell proliferation and cytokine secretion (Figure 5D). The CD3 6 chain is downregulated in vitro in the absence of L-arginine, resulting in decreased $\mathrm{T}$ cell proliferation (31). We observed that surface expression of the $\mathrm{CD} 3 \zeta$ chain on $\mathrm{CD}^{+}$and $\mathrm{CD} 8^{+} \mathrm{T}$ cells was significantly lower in both COVID-19 and influenza patients compared with HCs (Figure 5, E and F), suggesting that the $\mathrm{T}$ cells may have an impaired functional capacity. In summary, patients with COVID-19 had lower $\mathrm{T}$ cell counts and indications of impaired $\mathrm{T}$ cell function compared with $\mathrm{HCs}$.

Early M-MDSC frequency predicts peak disease severity. To evaluate the effect of early blood M-MDSC frequencies in COVID-19 on subsequent disease severity, we performed a proportional odds logistic regression analysis with the peak disease severity score as the primary outcome. Samples were selected from patients with COVID-19 for whom the M-MDSC frequency was measured within 2 weeks of disease onset, who had not already been admitted to the ICU, and who were not already in the recovery phase (Figure 6A). This yielded a crude OR of 1.43 (95\% CI, 1.07-2.18), indicating that M-MDSC frequency in the first 2 weeks from the onset of symptoms could potentially be a predictor of disease severity (Figure 6B). In an initial univariate ordinal regression, age but not sex had a significantly elevated OR (1.07 [95\% CI, 1.02-1.13] for age vs. 2.51 [95\% CI, 0.76-8.94] for sex). We then ran a model that included an adjustment for age (Figure 6B). An overview of the patients included in the analysis, the time points included in the model, and disease severity over time are shown in Figure 6C.

As shown above, M-MDSC frequencies were higher in COVID-19 patients with more severe disease (Figure 2C), and these patients were both predominantly male and significantly older than the COVID-19 patients with less severe disease (Table 1). Therefore, we assessed the association between M-MDSC frequency and sex and age and found that men had significantly higher frequencies of M-MDSCs (Figure $6 \mathrm{D})$ and that there was a significant correlation between age and M-MDSC frequency $\left(R=0.35, P=1.9 \times 10^{-5}\right.$; Figure $\left.6 \mathrm{E}\right)$.

In summary, early M-MDSC frequency is associated with subsequent disease severity and appear to be strongly associated with age and sex. 
Table 2. Baseline characteristics of patients with COVID-19 across disease severity levels

\begin{tabular}{|c|c|c|c|c|c|c|c|c|}
\hline \multirow{2}{*}{ Peak disease severity } & \multicolumn{2}{|c|}{ Mild } & \multicolumn{2}{|c|}{ Moderate } & \multicolumn{2}{|c|}{ Severe } & \multirow{2}{*}{$\begin{array}{c}\text { Fatal } \\
7\end{array}$} & \multirow{2}{*}{ Significance $^{A}$} \\
\hline & 1 & 2 & 3 & 4 & 5 & 6 & & \\
\hline$n(\%)$ & $13(8.8)$ & $6(4.1)$ & $10(6.8)$ & $48(33)$ & $19(13)$ & $39(27)$ & $12(8.2)$ & \\
\hline Age in yr, mean (range) & $44(24-72)$ & $60(41-72)$ & $56(46-78)$ & $55(24-76)$ & $57(42-74)$ & $61(25-77)$ & $66(52-78)$ & $P<0.001$ \\
\hline Female, $n(\%)$ & $8(62)$ & $4(67)$ & $4(40)$ & $10(21)$ & $4(21)$ & $5(13)$ & $3(25)$ & \\
\hline Onset to admission, ${ }^{\mathrm{B}}$ mean (SD) & - & $10.4(2.2)$ & $9.4(4.1)$ & $10.3(4.1)$ & $7.6(2.9)$ & $11.0(8.1)$ & $9.9(7.6)$ & NS \\
\hline \multicolumn{9}{|l|}{ Comorbidities } \\
\hline CCI, mean (SD) & $1(2)$ & $2(1)$ & $1(1)$ & $2(2)$ & $2(1)$ & $2(1)$ & $3(1)$ & $P \leq 0.05$ \\
\hline BMI, mean (SD) & $24.1(4.5)$ & $25.1(2.2)$ & $26.0(3.2)$ & $30.3(4.2)$ & $29.2(5.3)$ & $28.6(4.7)$ & $28.6(2.4)$ & $P<0.001$ \\
\hline Hypertension, $n$ (\%) & $1(8.3)$ & $0(0)$ & $2(20)$ & $20(42)$ & $8(42)$ & $15(38)$ & $9(75)$ & $P<0.01$ \\
\hline Diabetes, $n(\%)$ & $2(17)$ & $0(0)$ & $1(10)$ & $14(29)$ & $5(26)$ & $9(23)$ & $3(25)$ & NS \\
\hline $\mathrm{CRP}^{\mathrm{D}}$ mean (SD) & $6(10)$ & $102(54)$ & $137(143)$ & $161(87)$ & $236(114)$ & $288(106)$ & 364 (119) & $P<0.001$ \\
\hline WBC, ${ }^{\mathrm{E}}$ mean $(\mathrm{SD})$ & $4.7(1.6)$ & $6.0(1.9)$ & $6.8(4.9)$ & $7.2(2.6)$ & $7.6(2.7)$ & $9.7(5.7)$ & $10.8(5.3)$ & $P<0.01$ \\
\hline Neutrophils, ${ }^{\mathrm{E}}$ mean (SD) & $2.4(1.3)$ & $4.7(1.9)$ & $5.4(5.0)$ & $5.5(2.2)$ & $6.2(2.6)$ & $8.1(4.7)$ & $9.7(5.0)$ & $P<0.001$ \\
\hline Lymphocytes, ${ }^{\mathrm{E}}$ mean (SD) & $1.68(0.45)$ & $0.76(0.30)$ & $0.97(0.28)$ & $1.06(0.92)$ & $0.68(0.31)$ & $0.72(0.52)$ & $0.44(0.19)$ & $P<0.001$ \\
\hline Monocytes, ${ }^{\mathrm{E}}$ mean (SD) & $0.48(0.16)$ & $0.34(0.19)$ & $0.42(0.25)$ & $0.49(0.28)$ & $0.43(0.27)$ & $0.44(0.29)$ & $0.30(0.23)$ & NS \\
\hline Ct value, mean (SD) & $27(7)$ & $24(6)$ & $27(6)$ & $26(6)$ & $26(5)$ & $24(7)$ & $21(6)$ & NS \\
\hline Seroconversion, ${ }^{\mathrm{F}} n(\%)$ & $9(75)$ & $4(67)$ & $9(100)$ & $36(97)$ & $17(100)$ & $37(100)$ & $10(91)$ & $P<0.01$ \\
\hline
\end{tabular}

${ }^{A}$ One-way ANOVA or Fisher's exact test was performed to determine statistical significance. ${ }^{B}$ Days. ${ }^{C}$ Peak values: CRP; nadir values: Iymphocyte count, Ct value; WBC, neutrophil, and monocyte counts at the time point of the lowest lymphocyte count. ${ }^{\mathrm{D}} \mathrm{mg} / \mathrm{L}$. ${ }^{\mathrm{E}} 10^{9} \mathrm{cells} / \mathrm{L}$. ${ }^{\mathrm{F}} \mathrm{At}$ any sampling time point. Normal range: CRP $<3 \mathrm{mg} / \mathrm{L}$, WBC $3.5 \times 10^{9} / \mathrm{L}$ to $8.8 \times 10^{9} / \mathrm{L}$, lymphocytes $1.1 \times 10^{9} / \mathrm{L}$ to $3.5 \times 10^{9} / \mathrm{L}$, neutrophils $1.6 \times 10^{9} / \mathrm{L}$ to $5.9 \times 10^{9} / \mathrm{L}$, monocytes $0.2 \times 10^{9} / \mathrm{L}$ to $0.8 \times 10^{9} / \mathrm{L}$.

\section{Discussion}

Understanding the immunopathogenesis of COVID-19 is not only critical for optimal patient treatment and preventing fatal outcomes, but also for aiding in the development of specific therapies and vaccines. One potential player in immune activation during SARS-CoV-2 infection may be MDSCs, a subset of immune cells that in recent years have been intensively studied, by us and others, mainly in relation to cancer and vaccination (14-16, 18, 19, 32). Still, only limited information is available on how M-MDSCs influence disease severity during infection, including in COVID-19. In the present study, the distribution and function of M-MDSCs during COVID-19 were investigated over time and across disease severities in a comparatively large and clinically well-characterized cohort.

A major finding in the current study was the association between M-MDSC frequency and disease severity in patients with COVID-19. Downregulation of HLA-DR on monocytes has previously been shown in severe COVID-19, possibly reflecting an increase in M-MDSC frequencies, and is linked to high levels of IL- 6 and lymphopenia (33). We observed an increased frequency of blood M-MDSCs, during both COVID-19 and influenza, similar to what was previously observed in HIV-1 (17, 34). Although the COVID-19 and influenza patient cohorts were not completely comparable in terms of disease severity and time of sampling after symptom onset, it is still relevant to note that the expansion of M-MDSCs was also observed in another acute respiratory infection and that this finding was not unique to COVID-19. Furthermore, MDSCs are expanded during sepsis and are associated with poor outcomes (35).

Notably, in patients with influenza, M-MDSC frequencies were increased in the nasopharynx compared with the blood, indicating that M-MDSCs are recruited to the site of infection during influenza, as has been observed for other myeloid cells (36). In contrast, the frequency of M-MDSCs in the nasopharynx of patients with COVID-19 was similar to that in HCs. Although SARS-CoV-2 replication is initiated in the upper airways, it frequently progresses to the lower respiratory tract $(37,38)$ and may result in immune cell recruitment in the lower airways. However, we did not detect higher frequencies of M-MDSCs in ETAs than in NPAs. Nevertheless, ETAs do not necessarily contain cells from the alveoli, but rather cells from the trachea and bronchi. It is therefore possible that M-MDSCs are present even deeper down in the lungs and/or that the time points at which we obtained samples from the patients with COVID-19 were past the peak accumulation of M-MDSCs in the airways. Alternatively, M-MDSCs could differentiate into more macrophage-like cells at the site of inflammation, as seen after migration to tumor sites $(39,40)$, and would therefore not be identified with the flow cytometric staining panel we used. The migration of M-MDSCs from blood to the site of infection is supported by the fact that M-MDSCs in blood had upregulated levels of CD62L compared with respiratory M-MDSCs. $\mathrm{CD} 62 \mathrm{~L}$, or L-selectin, is involved in the extravasation of immune 
A

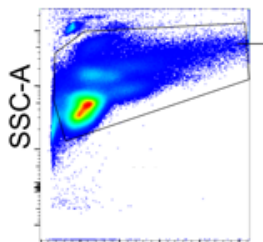

FSC-A

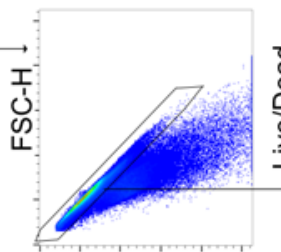

FSC-A
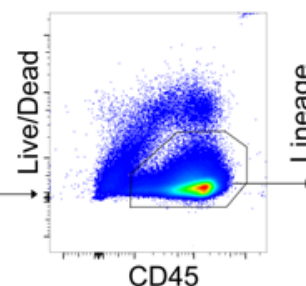

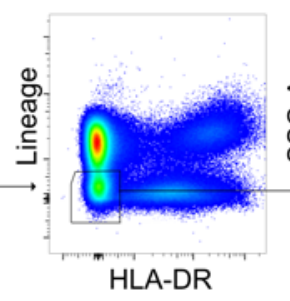

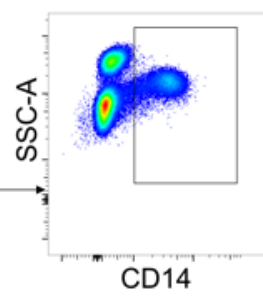

- COVID-19 - Mild COVID-19 - Moderate COVID-19 • Severe COVID-19 • Fatal COVID-19

- HC O Influenza o Mild influenza o Moderate influenza

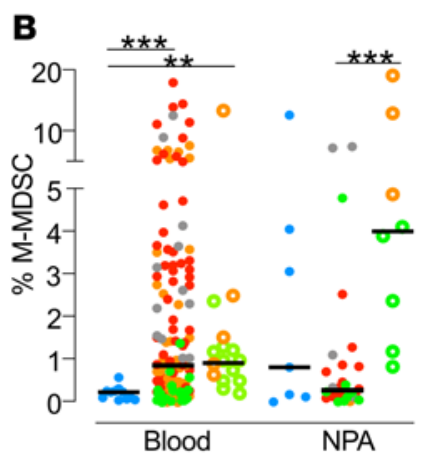

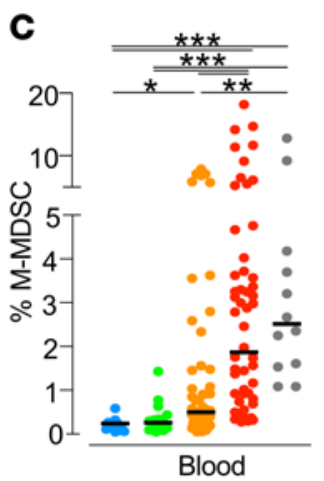

D

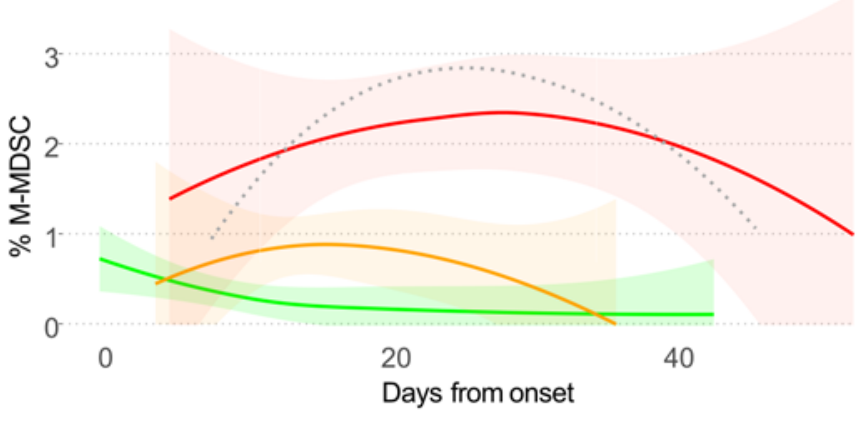

E
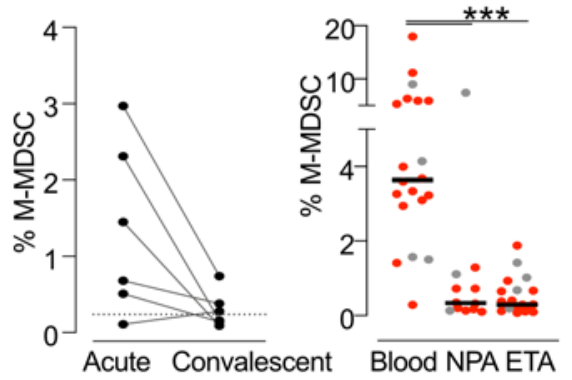

$\mathbf{F}$
G

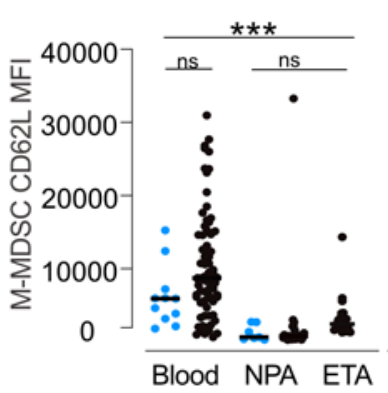

H

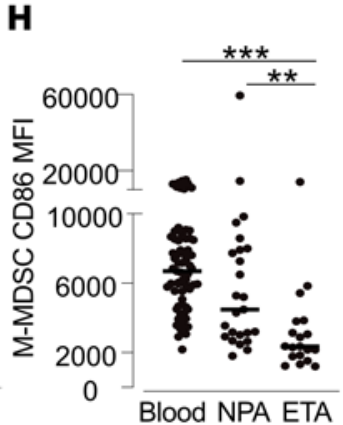

I

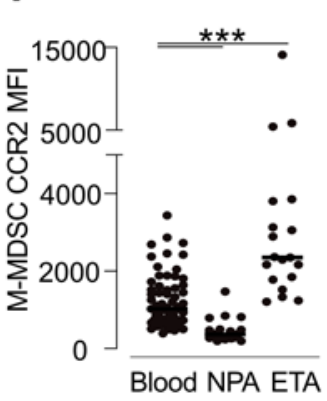

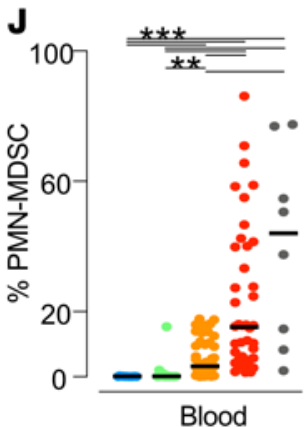

Figure 2. Frequency of respiratory and blood M-MDSCs in patients with CoVID-19, patients with influenza, and HCs. (A) Gating strategy to identify M-MDSCs by flow cytometry. From live, single CD45+ leukocytes, cells expressing lineage markers (CD3, CD19, CD20, CD56, CD66abce) and HLA-DR were excluded and CD14+ M-MDSCs identified. (B) M-MDSC frequency per live CD45+ cells in blood and NPAs. HCs (blue): $n=12$ (blood), $n=7$ (NPAs). Patients with influenza (open circles): $n=19$ (blood), $n=9$ (NPAs). COVID-19 patients (solid circles): $n=140$ (blood), $n=28$ (NPAs). The dots are color-coded according to peak disease severity. (C) Peak frequency of blood M-MDSCs per live CD45+ cells across disease severity. HCs (blue): $n=12$. Patients with COVID-19 (color-coded by peak disease severity): mild, $n=19$; moderate, $n=53$; severe, $n=56$; fatal, $n=12$. (D) Blood M-MDSC frequencies over time in patients with COVID-19: mild, $n=17$; moderate, $n=53$; severe, $n=56$; fatal, $n=12$. Line shows the locally estimated scatterplot smoothing (LOESS) with shaded $95 \% \mathrm{Cl}$ (fatal group wide CI, not presented). (E) Frequency of blood M-MDSCs in paired acute and convalescent samples from patients with COVID-19 ( $n=6$ ). (F) M-MDSC frequency in blood, NPA, and ETA samples from patients with severe (red, $n=16$ ) and fatal (gray, $n=4)$ COVID-19. (G-I) Surface expression of (G) CD62L, (H) CD86, and (I) CCR2 on M-MDSCs in blood, NPAs, and ETAs from HCs (blue, NPAs $n=7$, PBMCs $n=11$ ) and COVID-19 patients (black, NPAs $n=25$, ETAs $n=19$, PBMCs $n=69$ ). (J) Frequency of PMN-MDSCs of live CD45 cells in blood from patients with COVID-19. HCs: $n=12$. Patients with COVID-19: mild, $n=11$; moderate, $n=47$; severe, $n=42$; and fatal, $n=8$. (K) Frequency of blood PMN-MDSCs in paired acute and convalescent samples from patients with COVID-19 ( $n=6$ ). (B, C, and F-J) Comparisons of M-MDSC frequencies were performed using the nonparametric Kruskal-Wallis test with Dunn's post hoc multiple-comparison test. In the strip charts, group medians are presented as horizontal lines and individual patients as jitter points.

cells (41). Studies are ongoing to further address the kinetics and mechanism of myeloid cell migration in humans.

Glucocorticoid treatment may affect M-MDSCs and is therefore important to consider (42-45). In the current study, a relatively low proportion of the patients received glucocorticoids, and we did not observe any differences in M-MDSC frequencies between patients the patients who received glucocorticoid treatment and those who did not. It is therefore not likely that this is a significant confounder in our study.

Characterization of MDSCs is challenging because of the lack of unique cell-surface markers, and therefore functional analysis of the cells is critical to validate phenotypic identification $(15,20)$. We verified the suppressive capacity of M-MDSCs in patients with COVID-19 and found that HLA-D ${ }^{-}$CD14 ${ }^{+}$cells 
A

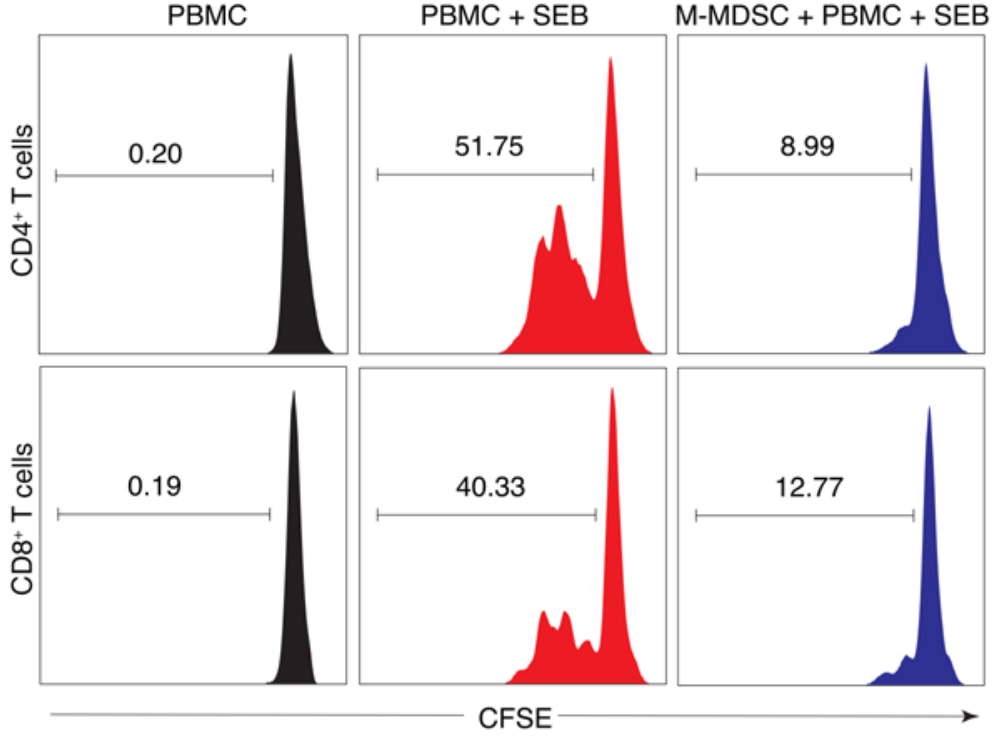

B
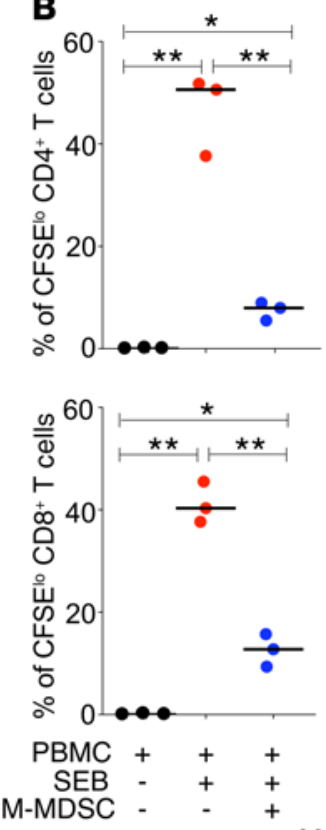

C
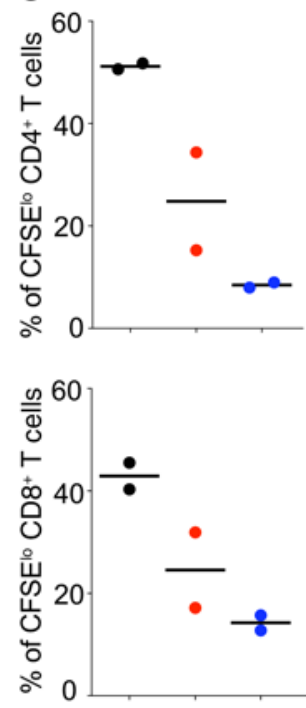

$\mathrm{PBMC}+++$

$\mathrm{SEB}+++$ M-MDSC +

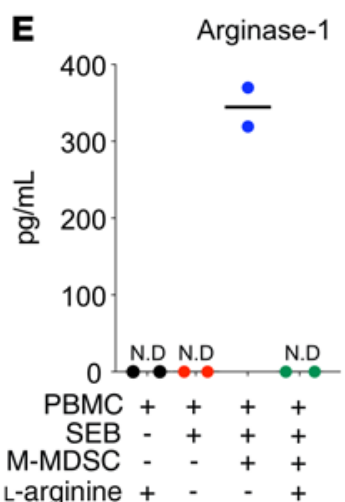

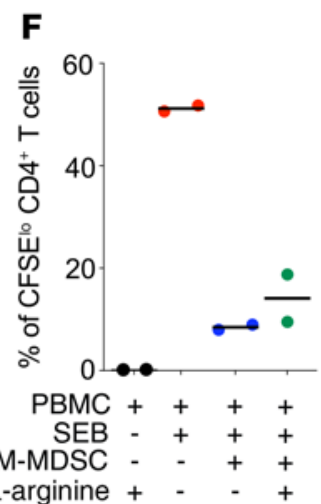

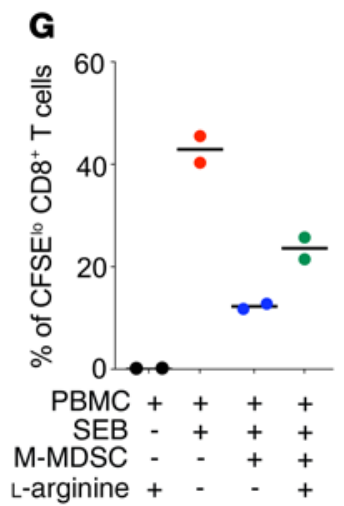

Figure 3. M-MDSCs isolated from patients with COVID-19 suppress T cell proliferation partly through the release of Arg-1. (A) Blood M-MDSCs isolated from patients with COVID-19 were cocultured with CFSE-labeled allogenic PBMCs in the presence of SEB for 3 days at a ratio of 1:2 (M-MDSCs/PBMCs). Histograms show representative $\mathrm{CD4} 4^{+}$and $\mathrm{CD} 8^{+} \mathrm{T}$ cell proliferation as assessed by CFSE dilution and flow cytometry. The numbers in the plots indicate the frequency of proliferating T cells. (B) Dot plots show the percentage of proliferating CD4+ and CD8 ${ }^{+} T$ cells with the median $(n=3) .{ }^{*} P \leq 0.05$ and ${ }^{* *} P<0.01$, by Wilcoxon signed-rank test. (C) Isolated M-MDSCs were cultured with CFSE-labeled allogenic PBMCs in the presence of SEB for 3 days. The M-MDSC/PBMC ratios were 1:5 and 1:2. Dot plots show the percentage of proliferating $C D 4^{+} \mathrm{T}$ and $\mathrm{CD} 8^{+}$cells with the median $(n=2)$. (D) Dot plots show IFN- $\gamma$ levels in supernatants from cell cultures with the median $(n=2)$. (E-C) Isolated M-MDSCs were cultured with CFSE-labeled allogenic PBMCs in the presence of SEB and L-arginine for 3 days. The M-MDSC/PBMC ratio was 1:2. (E) Dot plots show Arg-1 levels in supernatants from cell cultures with the median $(n=2)$. ND, not detectable. (F and $\mathbf{G})$ Dot plots show the percentage of proliferating (F) CD4 ${ }^{+}$T cells $(n=2)$ and $(\mathbf{C})$ CD8 ${ }^{+} T$ cells $(n=2)$ with the median.

isolated from these patients had a potent suppressive effect on $\mathrm{T}$ cells, demonstrating that the M-MDSCs identified by flow cytometry corresponded to suppressive and functionally active cells. Identifying PMN-MDSCs is even more challenging. The phenotypic identification of PMN-MDSCs varies among studies, and thus a functional analysis is also critical for these cells (19). Additionally, PMN-MDSCs are sensitive to cryopreservation (46), and all analyses require fresh cells. In the current study, this prevented us from performing a functional analysis of PMN-MDSCs using cryopreserved PBMCs. The focus of the current study was therefore on M-MDSCs.

Since Arg-1 was important for M-MDSC activity in vitro, we measured the levels in patients' plasma and NPAs. As expected, the patients with COVID-19 had increased plasma levels of Arg-1 compared with levels in HC plasma. We also found a connection between disease severity and Arg-1 levels in plasma, although there was no difference between patients with moderate disease and those who had a fatal outcome. Interestingly, Arg-1 levels were in general substantially higher in NPAs than in plasma for all cohorts, with no association with M-MDSC frequency in NPAs. It is known that Arg-1 is constitutively produced in the airways in bronchial epithelial cells, endothelial cells, myofibroblasts, and alveolar macrophages. Its function, however, is unknown. Arg-1 has been suggested to be involved in the regulation of NO and airway responsiveness and tissue repair (29). The association between M-MDSCs and Arg- 1 in the airways merits further examination. 

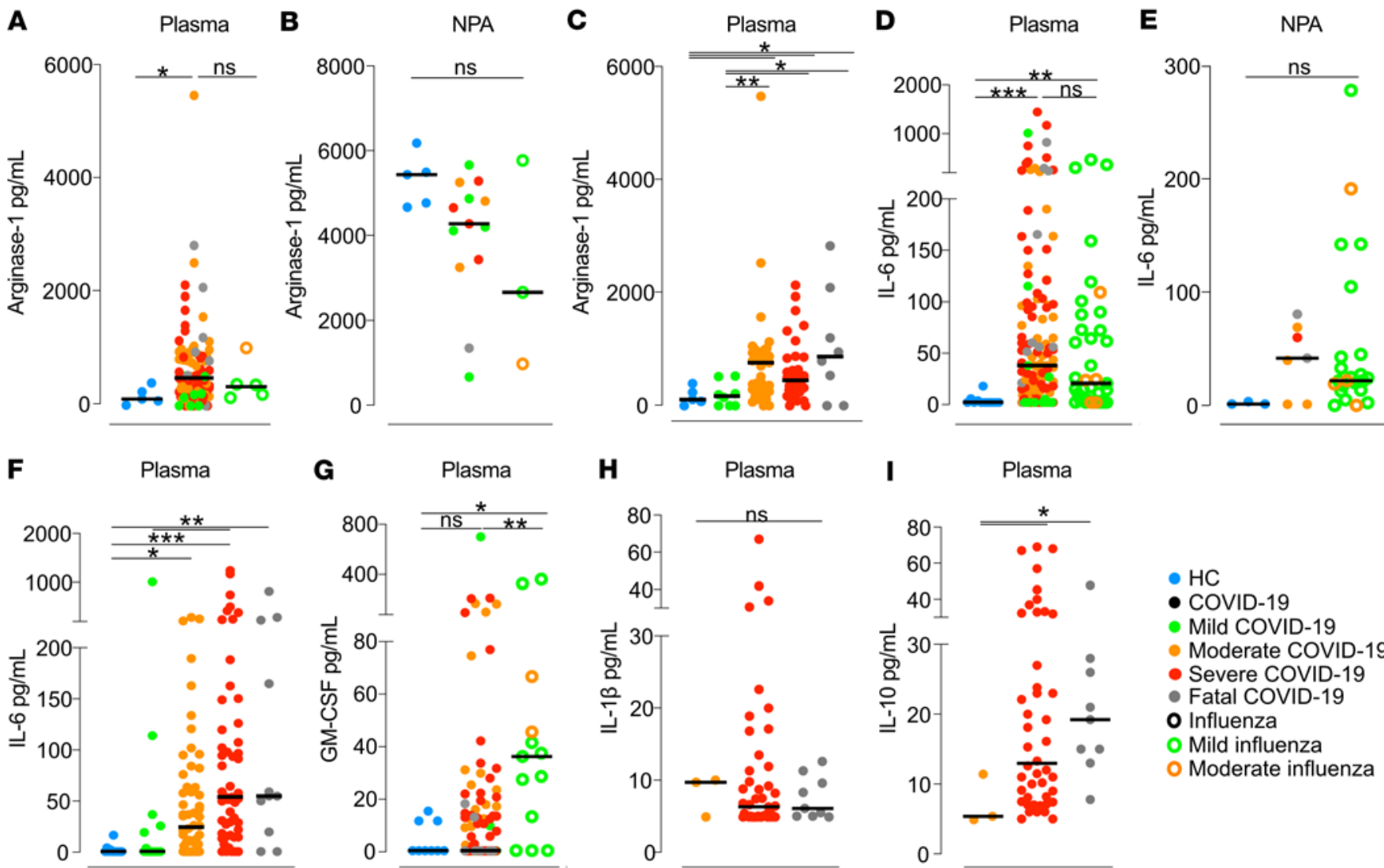

Figure 4. Levels of cytokines in blood and NPA from HCs, COVID-19 patients, and influenza patients. (A-C) Arg-1 was measured in (A) plasma, (B) NPAs, and (C) plasma across COVID-19 disease severities. HCs (blue points): $n=5$ (blood), $n=5$ (NPAs). Influenza patients (open circles): $n=6$ (blood), $n$ $=3$ (NPAs). Patients with COVID-19 (solid circles): $n=93$ (blood), $n=13$ (NPAs). Patients with COVID-19 (color-coded by peak disease severity): mild, $n=8$; moderate, $n=41$; severe, $n=36$; and fatal, $n=8$. (D-F) IL-6 was measured in (D) plasma, (E) NPAs, and (F) plasma across COVID-19 disease severities. HCs (blue): $n=11$ (blood), $n=3$ (NPAs). Patients with influenza (open circles): $n=37$ (blood), $n=24$ (NPAs). Patients with COVID-19 (solid circles): $n=133$ (blood), $n=7$ (NPAs). Patients with COVID-19: mild, $n=14$; moderate, $n=56$; severe, $n=52$; and fatal, $n=11$. (C) Comparison of GM-CSF levels in plasma from HCs and patients. HCs (blue): $n=9$. Patients with influenza (open circles): $n=13$. Patients with COVID-19 (solid circles, color-coded by peak disease severity): mild, $n=12$; moderate, $n=38$; severe, $n=48$; and fatal, $n=8$. (H) IL-1 $\beta$ and (I) IL-10 levels in plasma from patients with moderate-to-severe disease or a fatal outcome. COVID-19 patients: moderate, $n=3$; severe, $n=44$; and fatal, $n=9$. ${ }^{*} P \leq 0.05$, ${ }^{* *} P<0.01$, and ${ }^{* * *} P<0.001$. (A-I) Medians were compared using the nonparametric Kruskal-Wallis test. Post-hoc testing was carried out while controlling the FDR (A-C) or using Dunn's multiple-comparison test (D-I). In strip charts, the group medians are presented as horizontal lines and individual patients as jitter points.

Several factors, including IL-6 and GM-CSF, are involved in the expansion of M-MDSCs (14). Furthermore, IL-6 and IL-10 have been shown to be essential in inducing emergency myelopoiesis resulting in the expansion of an M-MDSC-like cell subset in severe COVID-19 (47). In line with previous studies $(12,33)$, we demonstrated a relationship between IL- 6 and COVID-19 disease severity. The high levels of IL- 6 could contribute to the generation of M-MDSCs, especially in patients with severe disease. In contrast to previous reports (48), plasma GM-CSF was not elevated in our COVID-19 cohort. Instead, higher levels of GM-CSF were detected in the influenza patient cohort. This could be explained by kinetics: the COVID-19 group had a longer duration of symptoms compared with the influenza group, and it is possible that the level of GM-CSF had already decreased. The lack of IFN- $\gamma$ in patients with COVID-19 is in line with other studies showing no or minor increases in IFN- $\gamma$ in COVID-19 patients and a reduced production of IFN- $\gamma$ in lymphocytes from these patients (49-51). However, the literature is not conclusive, and other studies have shown elevated levels of IFN- $\gamma$ in patients with COVID-19 $(2,52)$. IL-10 is also relevant with respect to MDSCs, since it can be produced by MDSCs $(14,18)$, and plasma levels of this cytokine increased with rising disease severity.

A decrease in the absolute numbers of both $\mathrm{CD} 4^{+}$and $\mathrm{CD} 8^{+}$ $\mathrm{T}$ cells, in line with the data presented here, has previously been demonstrated in patients with COVID-19 with severe disease, but the underlying mechanisms for the decrease are still unknown (53). One potential mechanism could be the downregulation of the $\mathrm{CD} 3 \zeta$ chain that results in impaired $\mathrm{T}$ cell proliferation. $\mathrm{CD} 3 \zeta$ chain downregulation on $\mathrm{T}$ cells has previously been observed in relation to MDSCs in several conditions including sepsis, hepatitis $\mathrm{C}$ infection, and gastric cancer (54-56). By establishing an immunosuppressive environment, M-MDSCs might prevent efficient immune activation and impede the development of specific adaptive responses required to clear the infection. We therefore speculate that expansion of M-MDSCs contributes to the immune imbalance described in COVID-19, possibly favoring disease progression. 
A

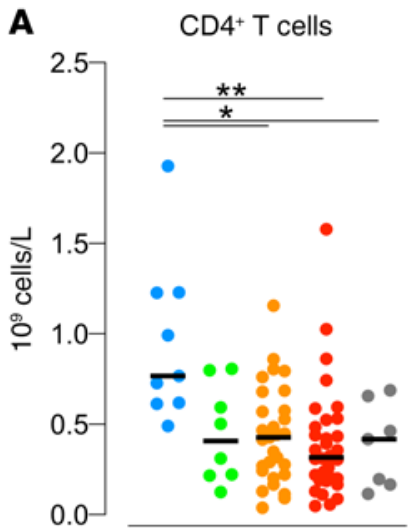

B

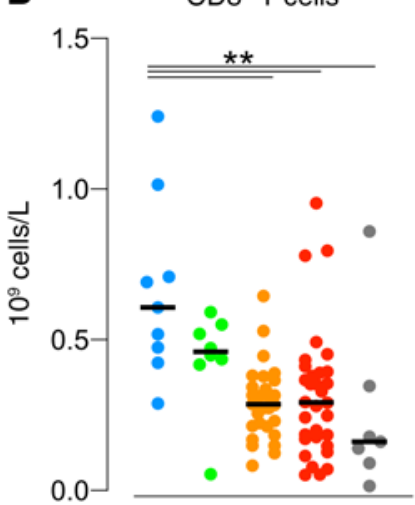

C

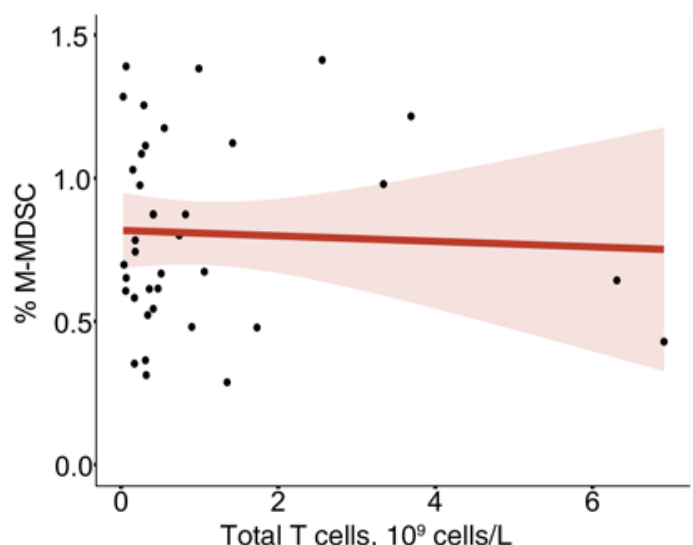

- HC • COVID-19 o Influenza

- Mild COVID-19 • Moderate COVID-19 • Severe COVID-19 • Fatal COVID-19

D

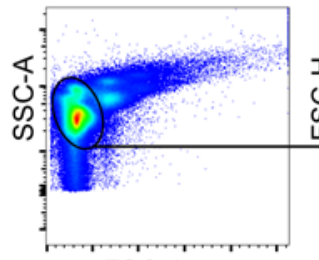

FSC-A

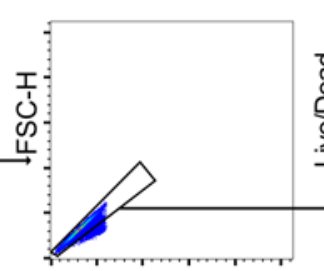

FSC-A

revere

- Fatal COVID-19 
A

COVID-19 patients with data on M-MDSC frequency, $n=140$

COVID-19 patients with symptom duration less than 2 weeks at time of sampling, $n=61$

COVID-19 patients not yet admitted to ICU, still in pre-peak/ peak phase, $n=41$

Ordinal logistic regression model to assess if early $\mathrm{M}$ MDSC frequency can predict disease outcome
B

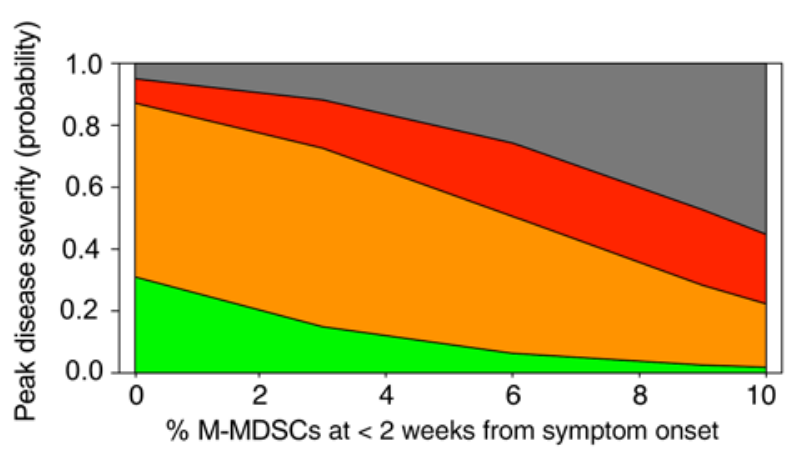

\begin{tabular}{|l|c|}
\hline & $\begin{array}{c}\text { M-MDSC frequency } \\
\text { OR }(95 \% \mathrm{Cl})\end{array}$ \\
\hline Crude OR & $1.43(1.07-2.18)$ \\
\hline Adj. for Age & $1.37(1.00-2.14)$ \\
\hline
\end{tabular}

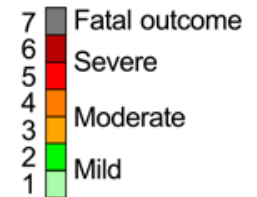

C

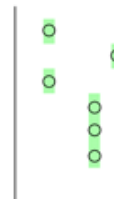

- Sample +Death

- COVID-19 • Mild COVID-19 • Moderate COVID-19 • Severe COVID-19 • Fatal COVID-19

D

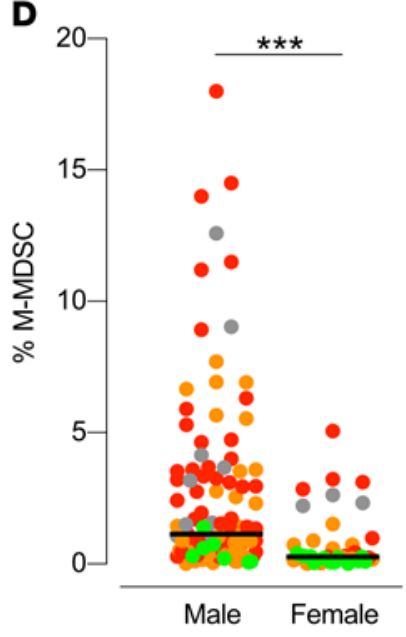

E

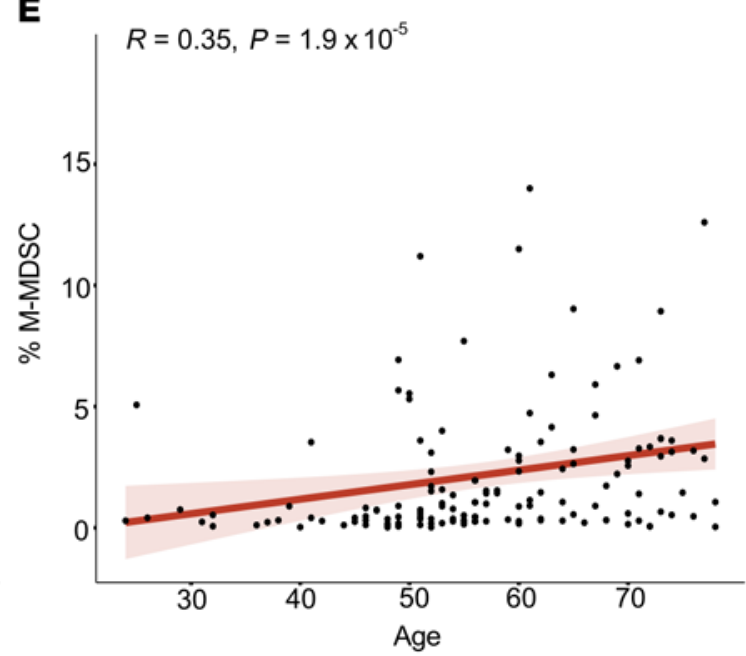


recruited from January 2019 through March 2020. A cohort of HCs (i.e., confirmed influenza A virus and SARS-CoV-2 negative by PCR) were recruited and sampled in the same way as the study patients.

The degree of respiratory failure was categorized daily according to the respiratory domain of the sequential organ failure assessment score (SOFA) (59). If arterial partial pressure of oxygen $\left(\mathrm{PaO}_{2}\right)$ was not available, peripheral transcutaneous hemoglobin saturation $\left(\mathrm{SpO}_{2}\right)$ was used instead and the modified SOFA score (mSOFA) was calculated (60). The fraction of inspired oxygen $\left(\mathrm{FiO}_{2}\right)$ estimation based on $\mathrm{O}_{2}$ flow was done in accordance with the Swedish Intensive Care Registry's definition (61). Patients were subsequently categorized on the basis of their peak respiratory SOFA or mSOFA value. The 5-point respiratory SOFA score was then extended with an additional level to distinguish patients admitted with mild disease from those with mild disease who were not admitted. Finally, fatal outcome was added as a seventh level, with a peak disease severity score of 6 prior to death in all but 2 patients who had scores of 4 and 5, respectively. Additionally, the resulting 7-point composite peak severity score was condensed into a classification consisting of mild (score of 1-2), moderate (score of 3-4), severe (score of 5-6), and fatal (score of 7) disease (Supplemental Tables 1 and 2).

Medical records were reviewed for clinical history, laboratory analyses, medications, previous diseases and comorbidities, and risk factors. The total burden of comorbidities was assessed using the CCI (62).

Collection of respiratory and blood samples. NPAs were collected from COVID-19 and influenza patients and HCs when possible, and ETAs were collected from intubated patients with COVID-19 in the ICU. Venous blood was collected in EDTA-containing tubes from all non-ICU patients and controls. For ICU patients, blood was pooled from heparin-coated blood gas syringes discarded in the previous 24 hours. For some ICU patients, additional venous blood samples were also collected in EDTA tubes. Routine clinical chemistry analysis was performed for all study participants including HCs. The samples were obtained from hospitalized patients at up to 4 time points, and discarded ICU patient material was collected at up to 10 time points.

Isolation of cells from blood and respiratory aspirates. NPA and ETA samples were centrifuged at $400 \mathrm{~g}$ for 5 minutes at room temperature (RT). Supernatant was collected and frozen at $-20^{\circ} \mathrm{C}$. Cells were washed with sterile PBS and mucus was removed using a $70 \mu \mathrm{m}$ cell strainer. Blood samples were centrifuged at $800 \mathrm{~g}$ for 8 minutes at RT. Plasma was collected and frozen at $-20^{\circ} \mathrm{C}$. The cellular fraction was diluted with sterile PBS, and PBMCs were isolated by density-gradient centrifugation at $900 \mathrm{~g}$ for 25 minutes at RT (without brake) using Ficoll-Paque Plus (GE Healthcare). Cell count and viability were assessed using trypan blue (MilliporeSigma) exclusion with an automated Countess cell counter (Invitrogen, Thermo Fisher Scientific). Cells were stained fresh for flow cytometric analysis. Excess PBMCs were cryopreserved in FBS (Gibco, Thermo Fisher Scientific) with $10 \%$ DMSO (MilliporeSigma) and stored in liquid nitrogen.

Flow cytometry. Cells were stained using Live/Dead Blue (Invitrogen, Thermo Fisher Scientific), incubated with human FcR blocking reagent (Miltenyi Biotec), and stained with antibodies against the following surface proteins: CD1c (AD5-8E7; Miltenyi Biotec), CD3 (SK7; BD), CD11c (B-Ly6; BD), CD14 (M5E2; BD), CD16 (3GE; BioLegend), CD19 (HIB19; BioLegend), CD20 (L27; BD), CD45 (HI30; BD), CD56 (HCD56; BD), CD62L (SK11; BD), CD66abce (TET2; Miltenyi Biotec), CD86 (2331; BD), CD123 (7G3; BD), CD141 (AD5-14H12; Miltenyi Biotec), CCR2 (K036C2; BioLegend), CCR7 (150503; BD), and HLA-DR
(TU36; Life Technologies, Thermo Fisher Scientific). If a sufficient number of cells were available, a second staining was performed using antibodies against CD3 (SP34-2; BD), CD4 (L200; BD), CD11c (B-ly6; BD), CD14 (M5E2; BD), CD16 (3G8; BD), CD19 (SJ25-C1; Thermo Fisher Scientific), CD45 (HI30; BD), CD56 (HCD56; BioLegend), CD66abce (TET2; Miltenyi Biotec), CD123 (7G3; BD), LOX-1 (15C4; BioLegend), and HLA-DR (L243; BioLegend). All stainings were performed at $4^{\circ} \mathrm{C}$ for 20 minutes. Cells were washed with PBS and fixed with $1 \%-2 \%$ paraformaldehyde.

The expression of $\mathrm{CD} 247$ (TCR $\zeta, \mathrm{CD} 3 \zeta$ ) on $\mathrm{CD} 4^{+}$and $\mathrm{CD} 8^{+} \mathrm{T}$ cells was evaluated by intracellular staining. Briefly, following surface staining with Live/Dead Blue (Invitrogen, Thermo Fisher Scientific), CD3 (SP34-2; BD), CD4 (L2O0; BD), and CD8 (SK1; BD), cells were fixed and permeabilized with permeabilization buffer (Thermo Fisher Scientific) and then stained with anti-CD247 (6B10.2; BioLegend) at $4^{\circ} \mathrm{C}$ for 20 minutes. Samples were acquired on an LSRFortessa flow cytometer (BD Biosciences). Data were analyzed using FlowJo software, version 10.5.3 (Tree Star). Absolute numbers of $\mathrm{CD}^{+}$and $\mathrm{CD} 8^{+} \mathrm{T}$ cells were calculated by multiplying the frequency of $\mathrm{T}$ cells among total lymphocytes obtained from flow cytometric data with the lymphocyte count from differential cell counts. If multiple $\mathrm{T}$ cell frequency counts were available from the same patient, the lowest $\mathrm{T}$ cell count was used. If the absolute lymphocyte count was missing, a value was linearly interpolated between existing values if no more than 7 days apart.

M-MDSC $T$ cell suppression assay. M-MDSCs (HLA-DR-CD14 ${ }^{+}$ cells) were purified from frozen PBMCs obtained from 3 patients with COVID-19, following a protocol developed by Lin et al. (16). HLA-DR ${ }^{+}$ cells were depleted using anti-HLA-DR microbeads and an LD column (Miltenyi Biotec). From the negative fraction, $\mathrm{CD} 14^{+}$cells were positively selected using anti-CD14 microbeads. MS columns and MACS separators (both from Miltenyi Biotec) were used for the cell sorting. Approximately 0.2 million M-MDSCs were obtained from 25 to 30 million PBMCs, with a viability of greater than $90 \%$ and a purity of greater than $85 \%$. In parallel, cryopreserved PBMCs from a buffy coat were thawed, and 4 million cells were labeled with CFSE (Thermo Fisher Scientific). The previously purified M-MDSCs were cocultured with 0.5 million of the CFSE-labeled PBMCs, at a ratio of $1: 2$ or 1:5, in the presence of $0.1 \mu \mathrm{g} / \mathrm{mL}$ SEB (MilliporeSigma) or $200 \mu \mathrm{g} / \mathrm{mL} \mathrm{L-ar-}$ ginine (MilliporeSigma). The cells were incubated for 3 days at $37^{\circ} \mathrm{C}$ in RMPI 1640 medium (MilliporeSigma) supplemented with 10\% FCS, 5 $\mathrm{mM} \mathrm{L}$-glutamine, and $100 \mathrm{U} / \mathrm{mL}$ penicillin and streptomycin (all from Invitrogen, Thermo Fisher Scientific). Supernatants were collected from cultures to measure secreted Arg-1 (Invitrogen, Thermo Fisher Scientific) and IFN- $\gamma$ (R\&D Systems) by ELISA. The cells were washed and surface-stained with CD3 (SK7), CD4 (OKT4), and CD8 (SK1) (all from BD Biosciences). Flow cytometry (LSRFortessa, BD Biosciences) was performed as described above, and T cell proliferation was measured by calculating the percentage of $\mathrm{CFSE}^{\text {lo }} \mathrm{T}$ cells.

Cytokine analysis. Cytokine levels were measured in plasma samples, NPA supernatants, and culture supernatants using ELISA. IL-6, GM-CSF, and IFN- $\gamma$ ELISAs were performed using DuoSet kits (R\&D Systems). The Arg-1 ELISA was performed using the Arginase-1 Human ELISA Kit (Thermo Fisher Scientific). IL-10 and IL-1 $\beta$ levels were analyzed at the Karolinska University Laboratory using Roche Cobas e602.

Plasma was also analyzed using a proximity extension assay (Olink Proteomics, ref. 63), performed at Stanford University according to the manufacturer's instructions. The 92-biomarker Inflammation panel 
(Olink Proteomics) was used. For this study, only data on IFN- $\gamma$ were included. Antibody pairs labeled with DNA oligonucleotides bound the target antigen and oligonucleotides were hybridized and extended by DNA polymerase. Subsequently, protein expression was measured by high-throughput real-time PCR. Protein levels are presented as normalized protein expression (NPX) values, an arbitrary unit in $\log _{2}$ scale. Values were calculated from inverted Ct values, with a high NPX value corresponding to a high protein concentration. Normalization of data was performed to minimize intra- and interassay variation.

Serology. Antibodies against the SARS-Cov-2 spike (S) trimer were assessed by ELISA. Recombinant proteins were received through the global health vaccine accelerator platform (GH-VAP) funded by the Bill \& Melinda Gates Foundation. Briefly, 96-well plates were coated with $100 \mathrm{ng} /$ well S protein. Plates were incubated with a selected duplicate dilution (1:50) of each plasma sample at ambient temperature for 2 hours. Detection was performed with a goat anti-human IgG HRP-conjugated secondary antibody (clone G18-145; BD Biosciences) followed by incubation with TMB substrate (BioLegend; catalog 421101) and stopped with a $1 \mathrm{M}$ solution of $\mathrm{H}_{2} \mathrm{SO}_{4}$. Absorbance was read at $450 \mathrm{~nm}+550 \mathrm{~nm}$ background correction using an ELISA reader. Data are reported as the average OD value of the 2 duplicates. An extensive analysis of respiratory and systemic antibody responses in the study cohort is available (64).

Statistics. Data analysis was performed using RStudio, version 1.2 (RStudio), GraphPad Prism, version 8.0 (GraphPad Software), and Microsoft Excel. Routine analyses excluding cytokines and flow cytometric data were assumed to have a standard distribution, and means were compared using either an independent Student's $t$ test or a 1-way ANOVA. Nominal patient characteristics were compared between groups using a Pearson $\chi^{2}$ test or Fisher's exact test depending on whether the expected count of any cell was above or below 5 . Cytokine and flow cytometric data were presumed to have a nonstandard distribution, and medians were thus compared using the Wilcoxon-Mann-Whitney $U$ or Kruskal-Wallis tests depending on the number of cohorts. Post hoc testing after the Kruskal-Wallis test was performed using Dunn's test of multiple comparisons or by controlling the FDR using Benjamini, Krieger, and Yekutieli's adaptive linear step-up procedure. Nonparametric comparisons of dependent data were performed using Wilcoxon's signed-rank test. For correlation analyses of continuous data, Spearman's rho was used. Finally, a proportional odds logistic regression model was constructed to evaluate the capacity of M-MDSC frequencies to predict disease severity. A 95\% significance level was used throughout the study, and all statistical tests were 2 tailed.

Missing daily severity score data were approximated by using the last-observation-carried-forward (LOCF) method. For flow cytometric data, the peak M-MDSC frequency and lowest T cell count were extracted. The peak value for routine laboratory analyses was extracted separately for each analysis except in the case of blood differential counts, in which all counts were extracted for the time point of lowest lymphocyte count. The ordinal logistic regression model was based on a subset of patients with M-MDSC frequencies who were sampled within 2 weeks of the onset of symptoms and before potential ICU admission. For patients who developed severe disease, only time points prior to peak disease severity were included, whereas in patients who developed at most mild-to-moderate disease, the time points occurring during recovery were excluded. In the event of multiple time points for 1 patient during that period, the first time point was used. An unadjusted OR was initially calculated individually for the capacity of M-MDSC frequency, age, and sex to predict peak disease severity, and statistically significant predictors were subsequently included in a multivariate proportional odds logistic regression model with the 4-level peak disease severity score as the primary outcome.

Study approval. The study was approved by the Swedish Ethics Review Authority (Etikprövningsmyndigheten, Uppsala, Sweden) and performed according to Declaration of Helsinki principles. Written informed consent was obtained from all patients and controls. For sedated patients, the denoted primary contact was asked about the presumed will of the patient and, if applicable, to give initial oral and subsequent signed written consent. When applicable, retrospective written consent was obtained from patients with nonfatal outcomes.

\section{Author contributions}

SFJ, SV, AL, K. Loré, NJ, AF, and ASS designed the study. SFJ, SV, MY, AC, IB, BÖ, MJL, IS, K. Lenart, and FH performed experiments. SFJ, RFJ, BÖ, EÅ, JS, MB, NJ, and AF recruited patients, obtained patient samples, and collected clinical data. JA provided relevant anonymized patient clinical data. SFJ, SV, MY, RFJ, AC, IB, MJL, RL, and $\mathrm{HM}$ analyzed data. SFJ, MY, RFJ, and MJL prepared figures. SFJ and ASS wrote the manuscript. All authors edited the manuscript.

\section{Acknowledgments}

We thank the patients and healthy volunteers who participated in this study. We would also like to thank the medical students and hospital staff for assistance with obtaining samples from patients and collection of clinical data. Experiments were performed in the Biomedicum BSL3 core facility at Karolinska Institutet. This work was supported by grants from the Swedish Research Council, the Swedish Heart-Lung Foundation, the Bill \& Melinda Gates Foundation, the Knut and Alice Wallenberg Foundation, and Karolinska Institutet. We would like to thank Deleah Pettie, Michael Murphy, Lauren Carter, and Neil P. King from the Department of Biochemistry at the University of Washington (Seattle, Washington, USA) and the Institute for Protein Design at the University of Washington for the production of viral proteins used in the antibody assay.

Address correspondence to: Anna Smed-Sörensen, Division of Immunology and Allergy, Department of Medicine Solna, Karolinska Institutet, Visionsgatan 4, BioClinicum J7:30, Karolinska University Hospital, 17164 Stockholm, Sweden. Email: anna.smed.sorensen@ ki.se. Phone: 46.8.617.768.29.

\footnotetext{
1. Zhou F, et al. Clinical course and risk factors for mortality of adult inpatients with COVID-19 in Wuhan, China: a retrospective cohort study. Lancet. 2020;395(10229):1054-1062.

2. Huang C, et al. Clinical features of patients infected with 2019 novel coronavirus in Wuhan,
}

China. Lancet. 2020;395(10223):497-506. 3. Wu C, et al. Risk factors associated with acute respiratory distress syndrome and death in patients with coronavirus disease 2019 pneumonia in Wuhan, China. JAMA Intern Med. 2020;180(7):934-943.
4. Ackermann M, et al. Pulmonary vascular endothelialitis, thrombosis, and angiogenesis in Covid-19. N Engl J Med. 2020;383(2):120-128.

5. Vabret $\mathrm{N}$, et al. Immunology of COVID-19: current state of the science. Immunity. 2020;52(6):910-941.

6. Baharom F, et al. Dendritic cells and monocytes 
with distinct inflammatory responses reside in lung mucosa of healthy humans. Jmmunol. 2016;196(11):4498-4509.

7. Vangeti S, et al. Human blood and tonsil plasmacytoid dendritic cells display similar gene expression profiles but exhibit differential type I IFN responses to influenza a virus infection. JImmunol. 2019;202(7):2069-2081.

8. Yu YR, et al. Flow cytometric analysis of myeloid cells in human blood, bronchoalveolar lavage, and lung tissues. Am J Respir Cell Mol Biol. 2016;54(1):13-24.

9. von Garnier C, et al. Anatomical location determines the distribution and function of dendritic cells and other APCs in the respiratory tract. JImmunol. 2005;175(3):1609-1618.

10. Desch AN, et al. Flow cytometric analysis of mononuclear phagocytes in nondiseased human lung and lung-draining lymph nodes. Am J Respir Crit Care Med. 2016;193(6):614-626.

11. Del Valle DM, et al. An inflammatory cytokine signature predicts COVID-19 severity and survival. Nat Med. 2020;26(10):1636-43.

12. Merad M, Martin JC. Pathological inflammation in patients with COVID-19: a key role for monocytes and macrophages. Nat Rev Immunol. 2020;20(6):355-362.

13. Sekine $\mathrm{T}$, et al. Robust $\mathrm{T}$ cell immunity in convalescent individuals with asymptomatic or mild COVID-19. Cell. 2020;183(1):158-168.

14. Gabrilovich DI, Nagaraj S. Myeloid-derived suppressor cells as regulators of the immune system. Nat Rev Immunol. 2009;9(3):162-174.

15. Cassetta L, et al. Deciphering myeloid-derived suppressor cells: isolation and markers in humans, mice and non-human primates. Cancer Immunol Immunother. 2019;68(4):687-697.

16. Lin A, et al. Rhesus macaque myeloid-derived suppressor cells demonstrate $\mathrm{T}$ cell inhibitory functions and are transiently increased after vaccination. J Immunol. 2018;200(1):286-294.

17. Wang J, et al. Effect of TLR agonists on the differentiation and function of human monocytic myeloid-derived suppressor cells. J Immunol. 2015;194(9):4215-4221.

18. Kumar V, et al. The nature of myeloid-derived suppressor cells in the tumor microenvironment. Trends Immunol. 2016;37(3):208-220.

19. Bronte V, et al. Recommendations for myeloid-derived suppressor cell nomenclature and characterization standards. Nat Commun. 2016;7:12150.

20. Bruger AM, et al. How to measure the immunosuppressive activity of MDSC: assays, problems and potential solutions. Cancer Immunol Immunother. 2019;68(4):631-644.

21. Schulte-Schrepping J, et al. Severe COVID-19 is marked by a dysregulated myeloid cell compartment. Cell. 2020;182(6):1419-1440.

22. Kvedaraite E, et al. Perturbations in the mononuclear phagocyte landscape associated with COVID-19 disease severity [preprint]. https:// doi.org/10.1101/2020.08.25.20181404. Posted on medRxiv August 25, 2020.

23. Agrati C, et al. Expansion of myeloid-derived suppressor cells in patients with severe coronavirus disease (COVID-19). Cell Death Differ. 2020;27(11):3196-3207.
24. Tang X, et al. Comparison of hospitalized patients with ARDS caused by COVID-19 and H1N1. Chest. 2020;158(1):195-205.

25. Petersen E, et al. Comparing SARS-CoV-2 with SARS-CoV and influenza pandemics. Lancet Infect Dis. 2020;20(9):e238-e244.

26. Condamine T, et al. Lectin-type oxidized LDL receptor-1 distinguishes population of human polymorphonuclear myeloid-derived suppressor cells in cancer patients. Sci Immunol. 2016;1(2):aaf8943.

27. Lang S, et al. Clinical relevance and suppressive capacity of human myeloid-derived suppressor cell subsets. Clin Cancer Res. 2018;24(19):4834-4844.

28. Marini $\mathrm{O}$, et al. Mature $\mathrm{CD} 10^{+}$and immature CD10- neutrophils present in G-CSF-treated donors display opposite effects on T cells. Blood. 2017;129(10):1343-1356.

29. Maarsingh $\mathrm{H}$, et al. Arginase and pulmonary diseases. Naunyn Schmiedebergs Arch Pharmacol. 2008;378(2):171-184.

30. Ribechini E, et al. Novel GM-CSF signals via IFN- $\gamma$ R/IRF-1 and AKT/mTOR license monocytes for suppressor function. Blood $A d v$. 2017;1(14):947-960.

31. Taheri F, et al. L-Arginine regulates the expression of the $\mathrm{T}$-cell receptor zeta chain (CD3zeta) in Jurkat cells. Clin Cancer Res. 2001;7(3 Suppl):958s-965s.

32. Cassetta L, et al. Differential expansion of circulating human MDSC subsets in patients with cancer, infection and inflammation. JImmunother Cancer. 2020;8(2):e001223.

33. Giamarellos-Bourboulis EJ, et al. Complex immune dysregulation in COVID-19 patients with severe respiratory failure. Cell Host Microbe. 2020;27(6):992-1000.e3.

34. Medina E, Hartl D. Myeloid-derived suppressor cells in infection: a general overview. J Innate Immun. 2018;10(5-6):407-13.

35. Schrijver IT, et al. Myeloid-derived suppressor cells in sepsis. Front Immunol. 2019;10:327.

36. Oshansky CM, et al. Mucosal immune responses predict clinical outcomes during influenza infection independently of age and viral load. Am J Respir Crit Care Med. 2014;189(4):449-462.

37. Hoffmann M, et al. SARS-CoV-2 cell entry depends on ACE2 and TMPRSS 2 and is blocked by a clinically proven protease inhibitor. Cell. 2020;181(2):271-280.e8.

38. Hou YJ, et al. SARS-CoV-2 reverse genetics reveals a variable infection gradient in the respiratory tract. Cell. 2020;182(2):429-446.

39. Corzo CA, et al. HIF-1 $\alpha$ regulates function and differentiation of myeloid-derived suppressor cells in the tumor microenvironment. J Exp Med. 2010;207(11):2439-2453.

40. Tcyganov E, et al. Plasticity of myeloid-derived suppressor cells in cancer. Curr Opin Immunol. 2018;51:76-82.

41. Springer TA. Traffic signals for lymphocyte recirculation and leukocyte emigration: the multistep paradigm. Cell. 1994;76(2):301-314.

42. $\mathrm{Lu} \mathrm{Y}$, et al. Glucocorticoid receptor promotes the function of myeloid-derived suppressor cells by suppressing HIF1 $\alpha$-dependent glycolysis. Cell Mol Immunol. 2018;15(6):618-629.

43. Wang Z, et al. Methylprednisolone alleviates multiple sclerosis by expanding myeloid-derived suppressor cells via glucocorticoid receptor $\beta$ and S100A8/9 up-regulation [published online October 23, 2020]. JCell Mol Med. https://doi. org $/ 10.1111 /$ jcmm. 15928 .

44. Hou Y, et al. High-dose dexamethasone corrects impaired myeloid-derived suppressor cell function via Ets1 in immune thrombocytopenia. Blood. 2016;127(12):1587-1597.

45. Moyes KW, et al. Effects of tumor grade and dexamethasone on myeloid cells in patients with glioma. Oncoimmunology. 2018;7(11):e1507668.

46. Kotsakis A, et al. Myeloid-derived suppressor cell measurements in fresh and cryopreserved blood samples. JImmunol Methods. 2012;381(1-2):14-22.

47. Reyes $\mathrm{M}$, et al. Induction of a regulatory myeloid program in bacterial sepsis and severe COVID-19 [preprint]. https://doi org/10.1101/2020.09.02.280180. Posted on bioRxiv September 2, 2020.

48. Wang J, et al. Cytokine storm and leukocyte changes in mild versus severe SARS-CoV-2 infection: review of 3939 COVID-19 patients in China and emerging pathogenesis and therapy concepts. J Leukoc Biol. 2020;108(1):17-41.

49. Chen G, et al. Clinical and immunological features of severe and moderate coronavirus disease 2019. J Clin Invest. 2020;130(5):2620-2629.

50. Varchetta $S$, et al. Unique immunological profile in patients with COVID-19 [published online October 15, 2020]. Cell Mol Immunol. https://doi. org/10.1038/s41423-020-00557-9.

51. Remy KE, et al. Severe immunosuppression and not a cytokine storm characterizes COVID-19 infections. JCI Insight. 2020;5(17):e140329.

52. Ghazavi A, et al. Cytokine profile and disease severity in patients with COVID-19. Cytokine. 2020;137:155323.

53. He R, et al. The clinical course and its correlated immune status in COVID-19 pneumonia. J Clin Virol. 2020;127:104361.

54. Darcy CJ, et al. Neutrophils with myeloid derived suppressor function deplete arginine and constrain $\mathrm{T}$ cell function in septic shock patients. Crit Care. 2014;18(4):R163.

55. Zeng QL, et al. Myeloid-derived suppressor cells are associated with viral persistence and downregulation of TCR $\zeta$ chain expression on CD8(+) $\mathrm{T}$ cells in chronic hepatitis $\mathrm{C}$ patients. Mol Cells. 2014;37(1):66-73.

56 . Wang L, et al. Increased myeloid-derived suppressor cells in gastric cancer correlate with cancer stage and plasma S100A8/A9 proinflammatory proteins. J Immunol. 2013;190(2):794-804.

57. Zheng Z, et al. Risk factors of critical and mortal COVID-19 cases: a systematic literature review and meta-analysis. JInfect. 2020;81(2):e16-e25.

58. Salminen A, et al. The role of myeloid-derived suppressor cells (MDSC) in the inflammaging process. Ageing Res Rev. 2018;48:1-10.

59. Vincent JL, et al. The SOFA (sepsis-related organ failure assessment) score to describe organ dysfunction/failure. On behalf of the Working Group on Sepsis-Related Problems of the European Society of Intensive Care Medicine. Intensive Care Med.1996;22(7):707-710.

60. Grissom CK, et al. A modified sequential organ failure assessment score for critical care triage. Disaster 
Med Public Health Prep. 2010;4(4):277-284.

61. Svenska Intensivvårdsregistret. SIR:s Riktlinje För Registrering Av SOFA. https://www.icuregswe. org/globalassets/riktlinjer/sofa_10.0.pdf. Updated February 2, 2018. Accessed September 1, 2020.

62. Charlson ME, et al. A new method of classifying prognostic comorbidity in longitudinal studies: development and validation. JChronic Dis. 1987;40(5):373-383.

63. Assarsson E, et al. Homogenous 96-plex PEA immunoassay exhibiting high sensitivity, specificity, and excellent scalability. PLoS One. 2014;9(4):e95192.

64. Cagigi A, et al. Airway antibodies wane rapidly after COVID-19 but B cell memory is generated across disease severity [preprint]. https://doi. org/10.1101/2020.11.25.20238592. Posted on medRxiv December 18, 2020. 